\title{
Postexercise hypotension and heart rate variability response after water- and land-based high-intensity interval exercise in prehypertensive obese men
}

\author{
Bhuwanat Sriton, Ratree Ruangthai, Jatuporn Phoemsapthawee* \\ Department of Sports Science and Health, Faculty of Sports Science, Kasetsart University, Nakhon Pathom, Thailand
}

A randomized crossover trial was carried out in prehypertensive obese men to compare postexercise hypotension and heart rate variability (HRV) following water-based and land-based high-intensity interval exercises (HIIEs). Nine prehypertensive obese participants, aged 23.6 \pm 2.4 years, were randomly assigned to one of three interventions: no-exercise control, HIIE with immersion up to the chest, or HIIE on dry land. In the evenings of three separate days, participants performed either of the interventions. Matched with exercise volume, both HIIEs composed of 5 repetitions of 30-sec sprints at maximum effort followed by a 4-min rest. Ambulatory blood pressure and HRV were measured before the interventions and over the 24-hr following period. Both HIIEs resulted in significant reductions of average 24-hr mean arterial pressure $(-6.7 \mathrm{mmHg})$. Notably, the water-based HIIE resulted in a significantly higher reduction of 24-hr systolic blood pressure (SBP) $(-9 \mathrm{mmHg})$ than the land-based HIIE, particularly at night, in addition to a significantly longer duration of postexercise hypotension. Finally, the water-based HIIE was more effective at restoring HRV during recovery. Our findings demonstrated postexercise hypotension following the HIIEs, particularly the water-based HIIE. During recovery, the water-based HIIE was remarkably effective at restoring HRV. These findings indicate that waterbased HIIE is more effective at reducing SBP and requires less recovery time than land-based HIIE in prehypertensive obese men.

Keywords: Ambulatory blood pressure, Heart rate variability, High-intensity interval training, Obesity, Postexercise hypotension

\section{INTRODUCTION}

Obesity is a growing global health concern, with evidence indicating a rapid rise in cardiovascular risk and an earlier onset of cardiovascular morbidity. Excess body mass triggers a cascade of pathophysiological events, resulting in the direct link between obesity and hypertension (Leggio et al., 2017). Hypertension is now the leading cause of cardiovascular disease (CVD), and treating hypertension imposes an exponentially greater economic burden. As a result, therapies for obesity-related hypertension include both weight loss and the reduction of other risk factors associated with obesity and CVD (Davy and Hall, 2004).

Regular exercise is critical in the prevention and treatment of nonpharmacological hypertension. The mechanisms underlying regular exercise's hypotensive benefits are complex and unknown (Keating et al., 2020; Pescatello et al., 2004). They do, however, appear to be associated with the prolonged postexercise hypotensive response (MacDonald, 2002; Liu et al., 2012). Several studies have shown that the blood pressure (BP) reductions experienced immediately after a single bout of exercise are comparable to those experienced after training (Hecksteden et al., 2013; Liu et al., 2012; Pescatello et al., 2015a; Pescatello et al., 2015b).

Furthermore, the magnitude and duration of the BP drop differ depending on the type and intensity of exercise. According to the hypertension management guidelines, the recommended practices primarily consist of moderate intensity and continuous exercises. In addition to these traditional modalities, high-intensity interval exercise (HIIE) is now recommended for hypertensive patients
${ }^{*}$ Corresponding author: Jatuporn Phoemsapthawee

(iD https://orcid.org/0000-0001-8859-3899

Department of Sports Science and Health, Faculty of Sports Science, Kasetsart

University, Nakhon Pathom 73140, Thailand

Email: jatuporn.w@ku.th

Received: November 15, 2021 / Accepted: December 12, 2021
This is an Open Access article distributed under the terms of the Creative Commons Attribution Non-Commercial License (https://creativecommons.org/licenses/by-nc/4.0/) which permits unrestricted non-commercial use, distribution, and reproduction in any medium, provided the original work is properly cited. 
(Pescatello et al., 2015b). It is becoming clear that a bout of HIIE is more effective at lowering BP than an isocaloric bout of moderate intensity and continuous exercises (Marçal et al., 2021; Pimenta et al., 2019; Ramirez-Jimenez et al., 2017; Sosner et al., 2016; Tucker et al., 2016).

While on-land HIIE has numerous benefits, participants must tolerate increased joint impact and the risk of acute muscle soreness or damage when performing weight-bearing exercise (Donnelly et al., 2009; Lopera et al., 2016). Due to the low load and mechanical stress placed on weight-bearing joints and muscles, water-based exercise is highly recommended for obese individuals (Donnelly et al., 2009). Additionally, it appears to have the benefit of lowering BP (Bocalini et al., 2017; Rodriguez et al., 2011; Sosner et al., 2016) and improving physical fitness (Lopera et al., 2016).

Both water- and land-based exercises have been shown to induce physiological adaptations, though the magnitudes of the effects vary (Bocalini et al., 2017; Rodriguez et al., 2011; Sosner et al., 2016). Water exercise has been shown to have a greater effect on sympathetic drive, catecholamine release, peripheral vascular resistance, and the vasopressin and renin-angiotensin systems than land exercise (Gabrielsen et al., 1996; Gabrielsen et al., 2000; Meredith-Jones et al., 2011; Reilly et al., 2003). Despite this, few studies have been conducted to determine the efficacy of postexercise hypotension following water-based exercise (Bocalini et al., 2017; Rodriguez et al., 2011; Sosner et al., 2016).

Heart rate variability (HRV) has been studied widely as a noninvasive method for estimating cardiac autonomic regulation (Task Force of the European Society of Cardiology and the North American Society of Pacing and Electrophysiology, 1996). Following exercise, the heart rate (HR) returns to pre-exercise levels due to changes in cardiac parasympathetic and sympathetic activity (Pober et al., 2004). However, obesity has been linked to a delay in the vagus nerve reactivation following exercise (El Agaty et al., 2017). Increased sinus node excitability as a result of decreased parasympathetic reactivation and increased sympathetic activity may explain the increased risk of sudden cardiac death following acute exercise (Albert et al., 2000). Thus, it is critical to assess the recovery dynamics associated with exercise. Although numerous studies have established that obese individuals have autonomic dysfunction at rest, little is known about the effect of obesity on cardiac autonomic recovery following exercise (Lopes et al., 2017).

As mentioned, there is a lack of data comparing postexercise hypotension and autonomic activity following water-based and land-based HIIE, especially in prehypertensive obese participants.
On the other hand, this information could be utilized to optimize exercise prescription in programs for obesity-related hypertension management. Thus, the purpose of this study was to compare the postexercise hypotension and HRV responses in prehypertensive obese men following water- and land-based HIIE.

\section{MATERIALS AND METHODS}

\section{Participants}

Prehypertensive, recreationally active obese men (20-29 years old) were recruited for this study. Of the 10 participants enrolled, nine (mean \pm standard deviation [SD]: age, 23.6 \pm 2.4 years; body mass index [BMI], $27.3 \pm 1.4 \mathrm{~kg} / \mathrm{m}^{2}$; percent body fat [\%BF], $27.4 \% \pm 5.1 \%$; peak oxygen consumption $\left[\mathrm{VO}_{2 \text { peak }}\right] 46 \pm 4.8 \mathrm{~mL} /$ $\mathrm{kg} / \mathrm{min}$; BP, $127.7 \pm 11.5 / 76.1 \pm 4.4 \mathrm{mmHg}$ ) (Table 1) completed the study. One participant was unable to complete the study due to lack of time. Participants were excluded if they currently, or had a history of ischemic heart disease, cerebrovascular disease, type 2 diabetes mellitus, advanced metabolic disease (e.g. chronic kidney disease), or if they were taking chronic medications known to affect BP. We also excluded participants who currently, or had a history of smoking. The study protocol was conducted according to the Declaration of Helsinki and approved by the Kasetsart University Research Ethics Committee (COA61/033).

\section{Study design}

This study employed a randomized, crossover, with repeated measures design. To avoid carryover effects, each participant completed two separate exercise protocols (water - and land-based HIIE) and a no-exercise condition in random order, with at least $72 \mathrm{hr}$

Table 1. Participants' characteristics $(\mathrm{n}=9)$

\begin{tabular}{lc}
\hline Variable & Mean \pm SD \\
\hline Age $(\mathrm{yr})$ & $23.6 \pm 2.4$ \\
Body mass $(\mathrm{kg})$ & $78.7 \pm 6.9$ \\
Height $(\mathrm{cm})$ & $169.8 \pm 5.5$ \\
$\mathrm{BMl}(\mathrm{kg} / \mathrm{m})$ & $27.3 \pm 1.4$ \\
$\% B F$ & $27.4 \pm 5.1$ \\
Resting HR (beats/min) & $76.2 \pm 10.6$ \\
Resting SBP $(\mathrm{mmHg})$ & $127.7 \pm 11.5$ \\
Resting DBP $(\mathrm{mmHg})$ & $76.1 \pm 4.4$ \\
Resting MAP $(\mathrm{mmHg})$ & $98.6 \pm 5.0$ \\
VO $_{2 \text { peak }}(\mathrm{mL} / \mathrm{kg} / \mathrm{min})$ & $46 \pm 4.8$ \\
\hline
\end{tabular}

SD, standard deviation; BMI, body mass index; \%BF, body fat percentage; HR, heart rate; SBP, systolic blood pressure; DBP, diastolic blood pressure; MAP, mean arterial pressure; $\mathrm{VO}_{2 \text { peak, }}$ peak oxygen consumption. 
between trials (Stanley et al., 2013). This design improved internal validity by allowing us to test our hypothesis and ensure that the results were applicable in practice. Participants completed baseline measurements over a 1-week period prior to beginning the trials. All tests were conducted in the early evening (between 4:00 and 6:00 p.m.), in a room maintained at a temperature of $25^{\circ} \mathrm{C}-26^{\circ} \mathrm{C}$. Ambulatory BP monitoring (ABPM) and HRV were measured prior to the study protocol session and $24 \mathrm{hr}$ after each trial to assess differences in BP and HRV variables between the experimental sessions. Additionally, participants were advised to abstain from stimulants such as caffeine and alcohol for $24 \mathrm{hr}$ prior to the tests, as well as from vigorous physical activity. They were also instructed to maintain their usual routines (i.e., diet, sleep) and to abstain from strenuous exercise during the week between experimental sessions.

\section{High-intensity interval exercise protocol}

Both exercise conditions started with a 10-min warm-up (jogging for 5 min at 60\%-70\% maximal HR (HRmax) and dynamic stretching for $5 \mathrm{~min}$ ). The HIIEs consisted of five 30-sec sprints at maximum speed, followed by 4 min of active recovery $(50 \%-$ $60 \%$ HRmax) (Angadi et al., 2015) in chest-deep water $\left(30^{\circ} \mathrm{C} \pm\right.$ $\left.1^{\circ} \mathrm{C}\right)$ and on land $\left(25^{\circ} \mathrm{C} \pm 1^{\circ} \mathrm{C}\right)$. The exercise sessions then ended with a 10-min cool down (walking for $5 \mathrm{~min}$ at $50 \%-60 \% \mathrm{HR}$ max and static stretching for $5 \mathrm{~min}$ ). Both HIIE modalities were similar in terms of exercise intensity and duration. Continuous HR monitoring (Polar H7, Polar Inc., Kempele, Finland) was used throughout exercise, and ratings of perceived exertion (RPE) were assessed at predetermined intervals using the Borg scale. Affective responses to exercise were assessed in-task and immediately following each bout of exercise, including their enjoyment of such exercise. After completing both HIIEs, participants were asked to indicate their preferred exercise.

\section{Preliminary visit}

Participants reported to the laboratory prior to the first experimental test day to have their anthropometrics, body composition, $\mathrm{BP}$, and $\mathrm{VO}_{2 \text { peak }}$ assessed. Body mass and body composition, including $\% \mathrm{BF}$, fat mass, and fat free mass, were measured using a bioimpedance analysis device (Inbody 720, Biospace Inc., Seoul, Korea) with participants wearing light clothing minus shoes. A standard stadiometer (Health O Meter Professional, Sunbeam Products Inc., Boca Raton, FL, USA) was employed to measure height minus shoes. BMI was determined by dividing weight by height squared. Resting BP and HR were measured using an au- tomated noninvasive BP monitor (Walk200b, Cardioline, Trento, Italy) between 08:00 and 10:00 a.m., with the participant seated according to standard protocols (Pickering et al., 2005).

$\mathrm{VO}_{2 \text { peak }}$ was determined using a modified Bruce protocol on a treadmill (T-2100 Treadmill, GE Medical Systems, Wauwatosa, WI, USA) in accordance with the exercise testing guidelines (Fletcher et al., 2001). Expired gas samples were collected on a breath-bybreath basis using a metabolic device (Vmax Encore Metabolic Cart, Vyaire Medical Inc., Yorba Linda, CA, USA), calibrated according to the manufacturer's instructions prior to each use. Oxygen consumption $\left(\mathrm{VO}_{2}\right)$, carbon dioxide production $\left(\mathrm{VCO}_{2}\right)$, and HR were continuously recorded and analyzed throughout the test. A rating of perceived exertion was obtained via the Borg scale (6$20)$ at the end of each work rate. An automatic sphygmomanometer (Tango M2, SunTech Medical Inc., Morrisville, NC, USA) was utilized to measure BP in the last 30 sec of each workload. $\mathrm{VO}_{2 \text { paak }}$ was defined as the average of the 3 highest consecutive 10-sec averages achieved during the test.

\section{4-Hr ambulatory BP monitoring}

$\mathrm{BP}$ was measured prior to participants beginning the study protocol sessions and 24-hr postsession. The measurements began at the same time of day and were conducted with an ABPM device (Walk200b, Cardioline) based on oscillometry with step deflation. The BP cuff was worn on the nondominant arm with the appropriate cuff size. Participants were instructed to carry out their normal daily activities, refrain from exercise, and to relax and straighten their arms during daytime ABPM recording. ABPM was analyzed and blinded to the trials by an examiner. Systolic BP (SBP) and diastolic BP (DBP) were classified using ABPM data according to average 24-hr, daytime, and nighttime periods. The daytime and nighttime periods were determined by when participants exited and entered their beds, respectively. The monitor was programmed to take BP recordings every 15 min during the day, and every $30 \mathrm{~min}$ at night. Individual BP measurements were examined for missing or erroneous data.

\section{HRV recordings and analysis}

The RR intervals were recorded by employing an electrocardiogram (ECG) (eMotion Faros device, Mega Electronics, Kuopio, Finland). The ECG was recorded twice: prior to and $24 \mathrm{hr}$ after the study protocol session, with all measurements taken on land to avoid water immersion-related changes. The RR intervals were analyzed via a software program using the recommendations of the Task Force of the European Society of Cardiology and the North 
American Society of Pacing and Electrophysiology (1996). Time and frequency-domain parameters together with nonlinear components of HRV were calculated. The time-domain parameters consisted of mean RR intervals, SD of normal RR intervals, and root mean square of successive differences (RMSSD). The frequency-domain parameters consisted of very low-frequency power (VLF power: $0.001-0.04 \mathrm{~Hz}$ ), low-frequency power (LF power: $0.04-$ $0.15 \mathrm{~Hz}$ ) and high-frequency power (HF power: $0.15-0.4 \mathrm{~Hz}$ ). Nonlinear HRV components, Poincaré plot SD perpendicular to the line of identity (SD1) and along the line of identity (SD2) were analyzed quantitatively by calculating the SDs from the RR interval data. The rhythmic activity of sinoatrial node is primarily regulated by the autonomous nervous system and varies in response to abrupt changes in BP. Therefore, it is widely accepted that HRV quantifies the heart's autonomic tone. Various HRV parameters were altered in response to sympathovagal balance changes (Malliani, 2005). In frequency- and time-domain analysis, the HF power, RMSSD and pNN50 may serve as indicators of vagal tone. The $\mathrm{LF} / \mathrm{HF}$ and $\mathrm{SD} 2 / \mathrm{SD} 1$ ratios were calculated to characterize the sympathovagal balance (Malliani, 2005).

\section{Affective response and enjoyment}

Affective response and enjoyment were assessed throughout the exercise sessions. Affective response employed a bipolar 11-point scale with numerical categories ranging from -5 to 5 . Each odd integer was paired with a verbal descriptor, with numerical categories ranging from "very bad" (-5), representing maximum displeasure/unpleasantness, to "neutral" (0), representing minimal pleasure, to "very good" $(+5)$, representing maximum pleasure (Hardy and Rejeski, 1989). Enjoyment employed a 7-point rating scale ranging from 1 (not at all) to 7 (extremely) to respond to the item: "Use the following scale to indicate how much you are enjoying this exercise session." All other integers on the scale were assigned modifiers (2 "very little", 3 "slightly", 4 "moderately", 5 "quite a bit", 6 "very much") (Stanley and Cumming, 2010).

\section{Muscle soreness}

After $24 \mathrm{hr}$ of HIIE sessions, participants were requested to rate their level of muscle soreness in the anterior thigh region after stepping up and down 4 times from a 40-cm-high box, thus recording their pain level on a scale immediately afterwards. Soreness was assessed using a verbal rating scale ranging from 0 to 10 , with 0 indicating no pain and 10 indicating the worst possible pain (e.g., "pain as bad as you can imagine" and "the worst pain imaginable") (Hawker et al., 2011).

\section{Statistical analysis}

The results are presented as mean $\pm \mathrm{SD}$ and, for inferential statistics, as mean \pm standard error of the mean. Sample size was based on the ability to detect a large effect (1.45) according to a previous report by Ramirez-Jimenez et al. (2017). As a consequence, it was decided upon to require $80 \%$ power at 0.05 significance. Hence, having at least 6 participants was required to complete the study. Normality was assessed by employing the Shapiro-Wilk test. If necessary, the data was transformed logarithmically prior to analysis of variance (ANOVA) in order to fulfill the criterion of normal distribution. Besides that, measurement outcomes were tested to determine whether they met the assumptions of normality, linearity, and homogeneity of variance. Outcome variable comparisons between the trials were performed through repeated measures, two-way ANOVA measures. Pairwise comparisons were performed by applying the least significant difference post hoc test. Within-group analyses were carried out with dependent $t$-test. Cohen effect size (ES) was calculated between each pair of measurements, as previously described (Sosner et al., 2016). Statistical significance was set at $P<0.05$. Statistical analyses were conducted by employing IBM SPSS Statistics ver. 26.0 (IBM Co., Armonk, NY, USA).

\section{RESULTS}

Table 1 shows the baseline characteristics. Participants' BMI, SBP and DBP were within the ranges of obesity and prehypertension. Throughout the exercise sessions, participants in the waterand land-based HIIEs exercised at $93.7 \% \pm 5.5 \%$ HRmax and $96.4 \% \pm 5 \%$ HRmax, respectively $(P>0.05)$. During each session, RPE responses in the water- and land-based HIIE were also frequent (18.3 \pm 1.9 and $19.1 \pm 1.1$, respectively, $P>0.05)$. There was no statistically significant difference in exercise intensity or RPE between HIIE modalities. Aside from the intervention, levels of habitual physical activity did not change significantly over time - with no difference observed between trials. There were no significant changes in daily calories, fat, carbohydrates, protein, or sodium consumption. Furthermore, no adverse events occurred in any of the trials.

Table 2 summarizes the average ABPM results at baseline and succeeding each modality of HIIE. Postexercise hypotension was observed in both HIIE modalities. The average of 24-hr SBP was significantly reduced $(-9 \mathrm{mmHg}, \mathrm{ES}=1.08, P<0.01)$ in the waterbased HIIE, though not in the land-based HIIE when compared to baseline. Additionally, SBP was significantly lower in the water- 
Table 2. Ambulatory blood pressure measurements at baseline and during the $24 \mathrm{hr}$ following the HIIE sessions or the no-exercise control session

\begin{tabular}{|c|c|c|c|}
\hline Variable & CON & Land-based HIIE & Water-based HIIE \\
\hline \multicolumn{4}{|l|}{ Baseline } \\
\hline $\mathrm{SBP}(\mathrm{mmHg})$ & $127.7 \pm 11.5$ & $125.4 \pm 9.0$ & $129.1 \pm 9.1$ \\
\hline $\mathrm{DBP}(\mathrm{mmHg})$ & $76.1 \pm 4.4$ & $75.9 \pm 7.4$ & $76.2 \pm 7.1$ \\
\hline $\mathrm{MAP}(\mathrm{mmHg})$ & $93.3 \pm 4.4$ & $92.3 \pm 6.6$ & $93.8 \pm 4.8$ \\
\hline \multicolumn{4}{|l|}{ 24-Hr period } \\
\hline $\mathrm{SBP}(\mathrm{mmHg})$ & $124.8 \pm 5.2$ & $120.5 \pm 8.3$ & $120.2 \pm 7.2^{* *,+t}$ \\
\hline $\mathrm{DBP}(\mathrm{mmHg})$ & $72.2 \pm 5.1$ & $68.2 \pm 5.2^{* *,+, \pm}$ & $70.5 \pm 5.2^{*}$ \\
\hline $\mathrm{MAP}(\mathrm{mmHg})$ & $89.8 \pm 3.9$ & $85.6 \pm 5.2^{* *, \dagger}$ & $87.0 \pm 5.1^{* *,+}$ \\
\hline \multicolumn{4}{|l|}{ Nighttime period } \\
\hline $\mathrm{SBP}(\mathrm{mmHg})$ & $116 \pm 6.8$ & $112.7 \pm 8.2^{* *}$ & $109 \pm 8.2^{* *,+1}$ \\
\hline $\mathrm{DBP}(\mathrm{mmHg})$ & $64.8 \pm 5.6$ & $60.2 \pm 6.3^{* *}$ & $62.0 \pm 4.3^{* *}$ \\
\hline $\mathrm{MAP}(\mathrm{mmHg})$ & $81.9 \pm 5.1$ & $77.8 \pm 6.2^{* *}$ & $77.7 \pm 4.9^{* *}$ \\
\hline \multicolumn{4}{|l|}{ Daytime period } \\
\hline $\mathrm{SBP}(\mathrm{mmHg})$ & $130.3 \pm 6.5$ & $128.8 \pm 9.7$ & $127 \pm 7.4$ \\
\hline $\mathrm{DBP}(\mathrm{mmHg})$ & $74.4 \pm 7.6$ & $73.5 \pm 6.9^{*}$ & $74.2 \pm 6.0$ \\
\hline $\mathrm{MAP}(\mathrm{mmHg})$ & $93 \pm 6.1$ & $91.9 \pm 6.7$ & $91.6 \pm 6.0$ \\
\hline
\end{tabular}

Values are presented as mean \pm standard deviation.

CON, no-exercise control; HIIE, high-intensity interval exercise; SBP, systolic blood pressure; DBP, diastolic blood pressure; MAP, mean arterial pressure.

${ }^{*} P<0.05$ and ${ }^{*} P<0.01$ to within-group comparison (baseline vs. post HIIE sessions). ${ }^{\dagger} P<0.05$ and ${ }^{\dagger t} P<0.01$ to between-group comparison (vs. CON). ${ }^{\ddagger} P<0.05$ to between-group comparison (land-based HIIIE vs. water-based HIIE).

based HIIE than in the no-exercise control condition over the 24-hr period ( $\mathrm{ES}=0.73, P<0.01)$. When compared to baseline, the average of 24-hr DBP in the land-based HIIE $(-7.7 \mathrm{mmHg}$, $\mathrm{ES}=1.2, P<0.01)$ and the water-based HIIE $(-5.6 \mathrm{mmHg}$, ES= $0.92, P<0.05)$ was significantly reduced. Additionally, the average of 24-hr DBP was significantly lower in the land-based HIIE than in the no-exercise control condition $(\mathrm{ES}=0.78, P<0.05)$ and in the water-based HIIE (ES $=0.44, P<0.05)$. The average of 24-hr mean arterial pressure (MAP) was similarly reduced following both the water - and land-based HIIEs $(-6.7 \mathrm{mmHg}, \mathrm{ES}=1.37$, $P<0.01$ and $-6.7 \mathrm{mmHg}, \mathrm{ES}=1.11, P<0.01$, respectively). The average of 24-hr MAP was also significantly lower in the water and land-based HIIEs than in the control condition with no exercise (ES $=0.64, P<0.05$ and $\mathrm{ES}=0.91, P<0.05$, respectively).

The average of SBP, DBP, and MAP were significantly lowered during the nighttime period following the land-based HIIE $(-12.7 \mathrm{mmHg}, \mathrm{ES}=1.48, P<0.01 ;-15.7 \mathrm{mmHg}, \mathrm{ES}=2.28, P<$ $0.01 ;-14.6 \mathrm{mmHg}, \mathrm{ES}=2.26, P<0.01$, respectively) and the water-based HIIE $(-20.1 \mathrm{mmHg}, \mathrm{ES}=2.32, P<0.01 ;-14.2 \mathrm{mmHg}$, $\mathrm{ES}=2.42, P<0.01 ;-16.1 \mathrm{mmHg}, \mathrm{ES}=3.32, P<0.01$, respectively), when compared to baseline. Only the water-based HIIE reduced SBP significantly (ES $=0.93, P<0.01$ ) when compared to the no-exercise control condition (Table 2).

Fig. 1 summarizes the changes in 24-hr ABPM using the traditional averaged BP intervals of $24 \mathrm{hr}$, daytime (06.00-22.00), and nighttime (22.00-06.00). After the first hour and the 7th to 12th hr following the HIIE sessions, SBP decreased significantly in the water- and land-based HIIEs compared to baseline. Only the land-based HIIE significantly decreased SBP in the 6th hr (ES= $0.73, P<0.05)$ compared to baseline. Meanwhile, only the waterbased HIIE significantly decreased SBP in the $14 \mathrm{th} \mathrm{hr}(\mathrm{ES}=1.55$, $P<0.01)$ in comparison to baseline. SBP was significantly lower in the first hour following the land-based HIIE compared to the no-exercise control $(\mathrm{ES}=1.25, P<0.05)$ and the water-based HIIE sessions ( $\mathrm{ES}=0.64, P<0.05$ ). Meanwhile, $\mathrm{SBP}$ was significantly lower in the 8th hr following the water-based HIIE than in the no-exercise control condition ( $\mathrm{ES}=1.32, P<0.05$ ). Moreover, $\mathrm{SBP}$ was significantly lower $12 \mathrm{hr}$ subsequent to the water-based HIIE than in the no-exercise control ( $\mathrm{ES}=1.38, P<0.01$ ) or the landbased HIIE sessions (ES=0.89, $P<0.05$ ) (Fig. 1). Although there was no significant difference in DBP between the experimental sessions following 24-hr ABPM assessments, DBP decreased significantly in the water- and land-based HIIEs after the 6th to 14th hr compared to baseline. Only the land-based HIIE exhibited a statistically significant reduction in DBP in the 15th hr (ES = $0.95, P<0.05$ ) compared to baseline (Fig.1).

Table 3 shows the average of the 24-hr HRV following the HIIE sessions or the no-exercise control condition. No significant differences were observed in any HRV variables between the water- and land-based HIIEs. After the water- and land-based HIIEs, mean $\mathrm{HR}$ (ES $=0.6, P<0.01$ and $\mathrm{ES}=0.63, P<0.05$, respectively) and $\mathrm{SD} 2 / \mathrm{SD} 1$ (ES $=0.37, P<0.05$ and $\mathrm{ES}=0.64, P<0.01$, respectively) were significantly higher than in the no-exercise control condition. The water- and land-based HIIEs resulted in significantly lower RR intervals ( $\mathrm{ES}=0.53, P<0.01$ and $\mathrm{ES}=0.65, P<0.05$, respectively), $\mathrm{pNN} 50$ (ES $=0.47, P<0.01$ and $\mathrm{ES}=0.72, P<0.01$, respectively), RMSSD (ES $=0.35, P<0.05$ and $\mathrm{ES}=0.77, P<0.01$, respectively), $\ln \mathrm{HF}(\mathrm{ES}=0.54, P<0.01$ and $\mathrm{ES}=0.8, P<0.01$, respectively), $\operatorname{lnLF}(\mathrm{ES}=0.44, P<0.01$ and $\mathrm{ES}=0.75, P<0.01$, respectively), $\operatorname{lnVLF}(\mathrm{ES}=0.57, P<0.05$ and $\mathrm{ES}=0.67, P<0.05$, respectively), $\operatorname{lnTP}(\mathrm{ES}=0.57, P<0.05$ and $\mathrm{ES}=1, P<0.01$, respectively), and $\mathrm{SD} 1$ (ES $=0.35, P<0.05$ and $\mathrm{ES}=0.76, P<0.01$, respectively). Only the land-based HIIE produced a significantly greater $\mathrm{LF} / \mathrm{HF}$ ratio than the control condition $(\mathrm{ES}=0.67, P<0.05)$.

There were no noteworthy differences in any of the HRV variables between the water- and land-based HIIEs at night or during the day. At nighttime, the water- and land-based HIIEs signifi- 

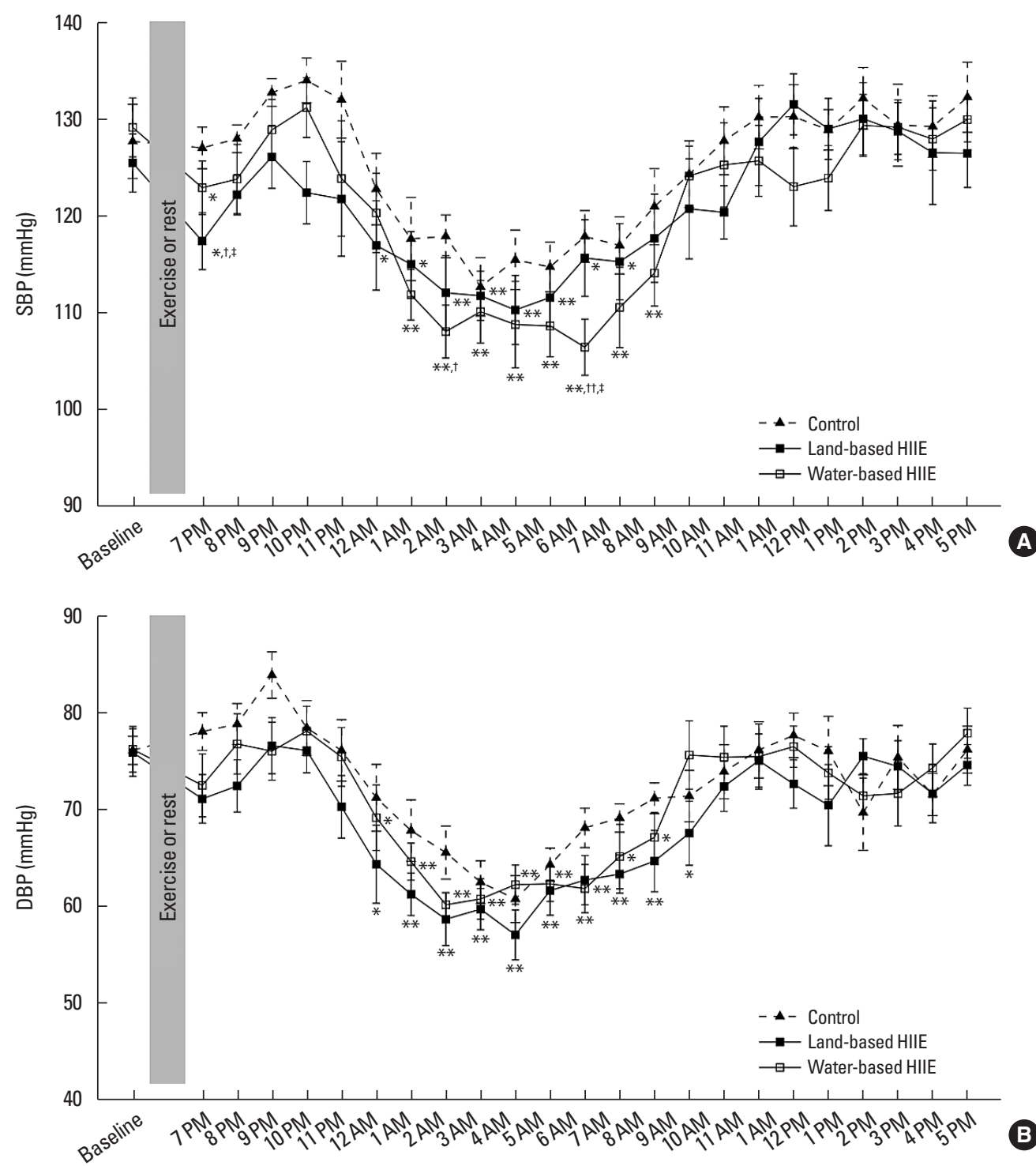

Fig. 1. Systolic blood pressure (SBP; A) and diastolic blood pressure (DBP; B) response during the $24 \mathrm{hr}$ following the HIIE sessions or the no-exercise control session. Data are expressed as mean \pm standard error of the mean. HIIE, high-intensity interval exercise. ${ }^{*} P<0.05$ and ${ }^{* *} P<0.01$ to within-group comparison (baseline vs. post HIIE sessions). ${ }^{\dagger} P<0.05$ and ${ }^{\dagger \dagger} P<0.01$ to between-group comparison (vs. CON). ${ }^{\ddagger} P<0.05$ to between-group comparison (land-based HIIE vs. water-based HIIE).

cantly reduced $\mathrm{RR}$ intervals $(\mathrm{ES}=0.48, P<0.01$ and $\mathrm{ES}=0.69$, $P<0.05$, respectively), $\mathrm{pNN} 50$ (ES $=0.39, P<0.05$ and $\mathrm{ES}=0.67$, $P<0.05$, respectively), $\operatorname{lnLF}$ ( $\mathrm{ES}=0.33, P<0.05$ and $\mathrm{ES}=0.5$, $P<0.05$, respectively), and $\operatorname{lnTP}(\mathrm{ES}=0.44, P<0.05$ and $\mathrm{ES}=0.88$, $P<0.05$, respectively) compared to the no-exercise control condition. Only the land-based HIIE significantly decreased RMSSD $(\mathrm{ES}=0.88, P<0.05), \operatorname{lnHF}(\mathrm{ES}=0.72, P<0.05), \operatorname{lnVLF}(\mathrm{ES}=1$, $P<0.05)$, and $\mathrm{SD} 1(\mathrm{ES}=0.88, P<0.05)$ compared to the no-exercise control condition. At daytime, in comparison to a no-exercise control condition, only the land-based HIIE significantly increased the $\mathrm{LF} / \mathrm{HF}$ ratio $(\mathrm{ES}=0.77, P<0.05)$ (Table 3).

In-task affective responses decreased over time in the water and land-based HIIEs. While the first two measurements were comparable across the HIIE sessions, the final three measurements were consistently different $(\mathrm{ES}=0.83, P<0.05, \mathrm{ES}=0.86, P<0.01$ and $\mathrm{ES}=0.77, P<0.05$, respectively), with the water-based HIIE session producing more positive affective responses than the landbased HIIE session (Fig. 2A). In both HIIE modalities, enjoyment while performing the tasks decreased over time. While enjoyment was comparable across the HIIE sessions for the first three measure- 
Sriton B, et al. • Postexercise hypotension after water- vs. land-HIIE

Table 3. Heart rate variability measurements during the $24 \mathrm{hr}$ following the HIIE sessions or the no-exercise control session

\begin{tabular}{|c|c|c|c|c|c|c|c|c|c|}
\hline \multirow[b]{2}{*}{ Variable } & \multicolumn{3}{|c|}{ 24-Hr period } & \multicolumn{3}{|c|}{ Nighttime period } & \multicolumn{3}{|c|}{ Daytime period } \\
\hline & CON & $\begin{array}{l}\text { Land-based } \\
\text { HIIE }\end{array}$ & $\begin{array}{c}\text { Water-based } \\
\text { HIIE }\end{array}$ & CON & $\begin{array}{l}\text { Land-based } \\
\text { HIIE }\end{array}$ & $\begin{array}{c}\text { Water-based } \\
\text { HIIE }\end{array}$ & CON & $\begin{array}{l}\text { Land-based } \\
\text { HIIE }\end{array}$ & $\begin{array}{c}\text { Water-based } \\
\text { HIIE }\end{array}$ \\
\hline HR (beats/min) & $74.4 \pm 6.4$ & $78.4 \pm 6.4^{\dagger}$ & $78.6 \pm 7.5^{t+}$ & $60.1 \pm 5.4$ & $62.3 \pm 2.8$ & $62.6 \pm 5.2$ & $84.1 \pm 9.0$ & $87.5 \pm 10.3$ & $85.6 \pm 12.7$ \\
\hline R interval (msec) & $856.0 \pm 77.3$ & $809.7 \pm 64.1^{\dagger}$ & $813.4 \pm 82.4^{+t}$ & $1,034.0 \pm 98.2$ & $981.5 \pm 43.9^{\dagger}$ & $990.6 \pm 83.8^{t+}$ & $740.3 \pm 84.2$ & $712.1 \pm 90.9$ & $731.7 \pm 113.9$ \\
\hline SDNN (msec) & $197.1 \pm 32.0$ & $180.2 \pm 25.4$ & $189.2 \pm 45.1$ & $156.5 \pm 45.0$ & $118.9 \pm 25.6$ & $145.7 \pm 45.4$ & $120.5 \pm 32.4$ & $108.6 \pm 45.7$ & $106.0 \pm 37.3$ \\
\hline pNN50 (\%) & $26.6 \pm 8.4$ & $21.0 \pm 7.1^{t t}$ & $22.2 \pm 10.2^{t+}$ & $47.1 \pm 12.5$ & $39.3 \pm 10.7^{\dagger}$ & $41.9 \pm 13.8^{\dagger}$ & $12.6 \pm 7.0$ & $10.6 \pm 8.1$ & $13.1 \pm 12.7$ \\
\hline RMSSD (msec) & $68.1 \pm 19.5$ & $54.3 \pm 16.3^{\text {tt }}$ & $60.4 \pm 24^{\dagger}$ & $88.7 \pm 23.1$ & $70.4 \pm 18.0^{\dagger}$ & $80.2 \pm 29.7$ & $43.6 \pm 13.7$ & $37.6 \pm 20.7$ & $40.9 \pm 23.7$ \\
\hline $\operatorname{lnHF}\left(\mathrm{msec}^{2}\right)$ & $7.3 \pm 0.5$ & $6.9 \pm 0.5^{t+}$ & $7.0 \pm 0.6^{\dagger t}$ & $7.8 \pm 0.5$ & $7.4 \pm 0.6^{\dagger}$ & $7.6 \pm 0.6$ & $6.2 \pm 0.6$ & $5.8 \pm 0.8$ & $5.9 \pm 1.0$ \\
\hline $\operatorname{lnLF}\left(\mathrm{msec}^{2}\right)$ & $7.4 \pm 0.4$ & $7.1 \pm 0.4^{\dagger t}$ & $7.2 \pm 0.5^{\dagger t}$ & $7.5 \pm 0.6$ & $7.2 \pm 0.6^{\dagger}$ & $7.3 \pm 0.6^{\dagger}$ & $6.9 \pm 0.3$ & $6.8 \pm 0.5$ & $6.8 \pm 0.6$ \\
\hline $\operatorname{lnVLF}\left(\mathrm{msec}^{2}\right)$ & $8.8 \pm 0.3$ & $8.6 \pm 0.3^{\dagger}$ & $8.6 \pm 0.4^{\dagger}$ & $8.8 \pm 0.4$ & $8.4 \pm 0.4^{\dagger}$ & $8.6 \pm 0.5$ & $8.0 \pm 0.5$ & $7.8 \pm 0.6$ & $7.8 \pm 0.7$ \\
\hline $\operatorname{lnTP}\left(\mathrm{msec}^{2}\right)$ & $9.2 \pm 0.3$ & $8.9 \pm 0.3^{t t}$ & $9.0 \pm 0.4^{\dagger}$ & $9.3 \pm 0.4$ & $8.9 \pm 0.5^{\dagger}$ & $9.1 \pm 0.5^{\dagger}$ & $8.5 \pm 0.5$ & $8.2 \pm 0.6$ & $8.2 \pm 0.7$ \\
\hline LF/HF ratio & $1.1 \pm 0.3$ & $1.3 \pm 0.3^{\dagger}$ & $1.2 \pm 0.3$ & $0.8 \pm 0.3$ & $0.9 \pm 0.3$ & $0.8 \pm 0.3$ & $2.1 \pm 0.8$ & $2.8 \pm 1.0^{\dagger}$ & $2.7 \pm 1.0$ \\
\hline SD1 (msec) & $48.1 \pm 13.8$ & $38.4 \pm 11.5^{t \dagger}$ & $42.7 \pm 17.0^{\dagger}$ & $62.7 \pm 16.3$ & $49.8 \pm 12.8^{\dagger}$ & $56.7 \pm 21.0$ & $30.8 \pm 9.7$ & $26.6 \pm 14.6$ & $28.9 \pm 16.8$ \\
\hline SD2 (msec) & $274.4 \pm 44.4$ & $251.8 \pm 34.8$ & $263.9 \pm 62.3$ & $211.7 \pm 63.1$ & $160.6 \pm 34.3$ & $197.9 \pm 61.3$ & $167.5 \pm 45.2$ & $151.1 \pm 63.1$ & $146.9 \pm 50.7$ \\
\hline SD2/SD1 & $6.0 \pm 1.4$ & $6.9 \pm 1.4^{\dagger t}$ & $6.6 \pm 1.8^{\dagger}$ & $3.5 \pm 0.9$ & $3.3 \pm 0.5$ & $3.6 \pm 0.5$ & $5.6 \pm 1.2$ & $6.0 \pm 1.3$ & $5.7 \pm 1.5$ \\
\hline
\end{tabular}

Values are presented as mean \pm standard deviation.

HIIE, high-intensity interval exercise; CON, no-exercise control; HR, heart rate; SDNN, standard deviation of all RR intervals; pNN50, percentage of consecutive RR intervals that differ by > 50 msec; RMSSD, root mean square of differences of successive RR intervals; InHF, logarithm high frequency; InLF, logarithm low frequency; InVLF, logarithm very low frequency; InTP, logarithm total power; LF/HF ratio, ratio of absolute LF power to HF power; SD1, the standard deviation of the distance of each point from the $y=x$-axis; SD2, the standard deviation of each point from the $y=x+$ average RR interval; SD2/SD1, ratio of SD2 to SD1.

${ }^{\dagger} P<0.05$ and ${ }^{t+} P<0.01$ to between-group comparison (vs. CON)
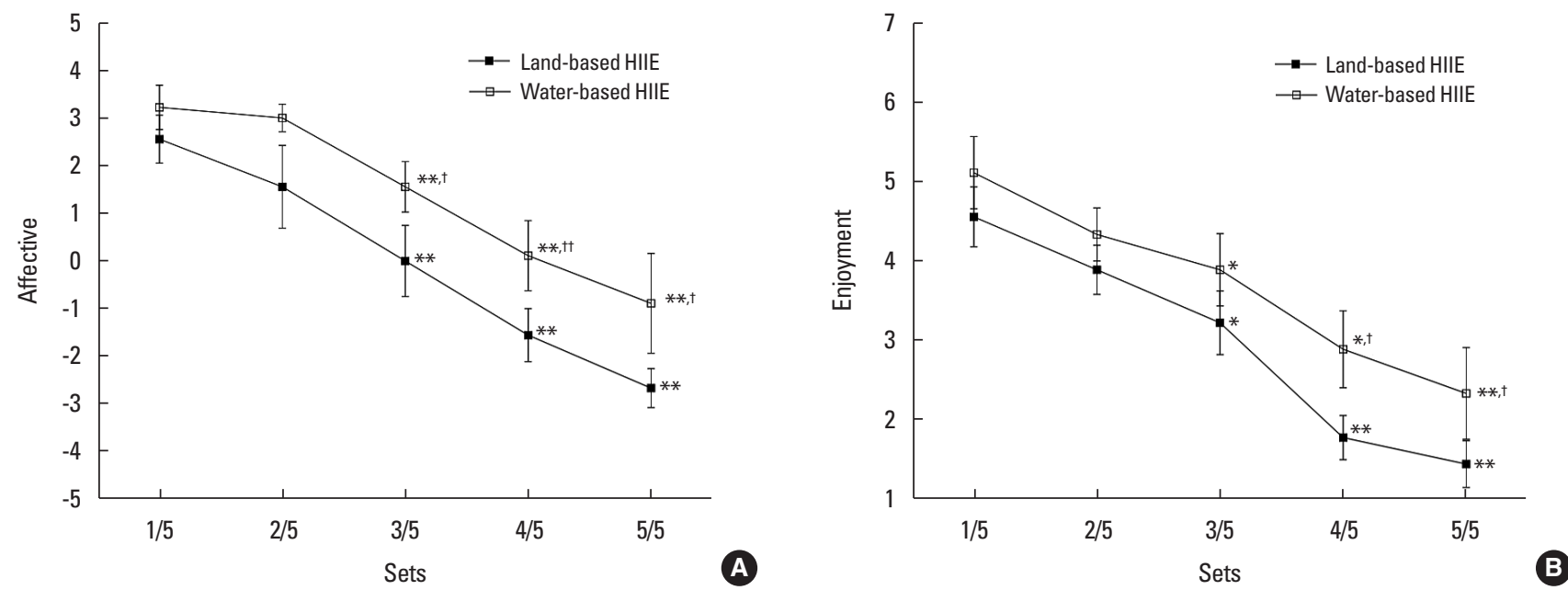

Fig. 2. Affective (A) and enjoyment (B) responses during the water- and land-based HIIE. Data are expressed as mean \pm standard error of the mean. ${ }^{*} P<0.05$ and ${ }^{* *} P<0.01$ to within-group comparison (vs. the 1 st set). ${ }^{\dagger} P<0.05$ and ${ }^{t \dagger} P<0.01$ to between-group comparison (land-based HIIE vs. water-based HIIE). HIIE, high-intensity interval exercise.

ments, it was significantly different for the final two $(\mathrm{ES}=0.92$, $P<0.05$ and $\mathrm{ES}=0.66, P<0.05$, respectively). The water-based HIIE session produced more positive enjoyment than the landbased HIIE session (Fig. 2B). Following $24 \mathrm{hr}$ of HIIE sessions, perceived pain intensity in the anterior thigh region was significantly lower in the water-based HIIE than on land $(1.1 \pm 1.5$ vs.
$3.3 \pm 2.6, \mathrm{ES}=0.99, P<0.05)$

\section{DISCUSSION}

Postexercise hypotension plays a critical role in the improvement of $\mathrm{BP}$ with exercise training, even if its mechanism is not well un- 
derstood. To our knowledge, this is the first study to investigate the effect of a single bout of water- and land-based HIIEs on the postexercise hypotension and HRV parameters of obese prehypertensive men. Our primary finding is that postexercise hypotension was detected in both water- and land-based HIIEs. Similar reductions in the average 24-hr MAP were observed following the HIIE modalities $(-6.7 \mathrm{mmHg})$. Notably, the water-based HIIE resulted in a pointedly greater reduction of SBP $(-9 \mathrm{mmHg})$ than the landbased HIIE, particularly at night. The water-based HIIE resulted in considerably longer durations of postexercise hypotension than the land-based HIIE, with reductions in nighttime SBP. Furthermore, we discovered that SBP decreased following the land-based HIIE, though for a shorter period of time in our study ( $1 \mathrm{hr}$ ).

There is very little known concerning the role of water-based HIIE in terms of postexercise hypotension and HRV parameters. The majority of studies to date have focused on moderate intensity and continuous exercises in water (Bocalini et al., 2017; Rodriguez et al., 2011). There is only one previous study focusing on water-based HIIE in prehypertensive and hypertensive older adults (Sosner et al., 2016). However, our findings demonstrated postexercise hypotension after $8 \mathrm{hr}$ of water-based HIIE, which is consistent with previous reports (Bocalini et al., 2017; Rodriguez et al., 2011; Sosner et al., 2016) despite slightly later than the time hypotension. The mechanisms underlying hypotension after exercise in water should be related to the aquatic environment. One possibility is that baroreceptor stimulation is influenced by hydrostatic pressure. Thus, hydrostatic pressure promotes venous return, resulting in increased cardiac output via increased end diastolic volume. The redistribution of blood volume during immersion may result in an increase in BP, stimulating the baroreceptor reflex. As a consequence, $\mathrm{BP}$ is continuously decreased for a number of hours following immersion (Meredith-Jones et al., 2011; Rodriguez et al., 2011). Additionally, hydrostatic pressure facilitates the transfer of fluid from peripheral to central organs and promotes diuresis, which results in a decrease in blood volume (Jimenez et al., 2010).

For another possibility, the decrease in BP may be related to decreased sympathetic nervous system activity and adaptation mechanisms in the cardiovascular system, such as decreased renin, angiotensin II, aldosterone, and atrial natriuretic peptide secretion (Barbosa et al., 2009; Meredith-Jones et al., 2011; Rodriguez et al., 2011). These hydrostatic pressure-activated mechanisms offer advantages over neuro-hormonal control of BP. Alternatively, the increased muscle mass activities in water-based exercises may result in increased production of vasodilators such as adenosine, po- tassium, lactate, nitric oxide (NO), and prostaglandin (Routledge et al., 2010). Additionally, the hypotensive effect is highly dependent on the intensity, mode, and duration of exercise (Jones et al., 2009; MacDonald et al., 2000). A greater nighttime drop in BP is associated with an increase in exercise intensity (Jones et al., 2009) which is critical for meaningful cardiovascular outcomes (Mancia et al., 2013). Although both exercise interventions were HIIE, nighttime SBP dipping occurred in just the water-based HIIE, and not in the land-based HIIE. Reduced SBP during the nighttime period has been reported to be related to sleep (Calhoun and Harding, 2010). Our data demonstrated that both HIIEs had no effect on sleep disturbances as HR, LF/HF, and SD2/SD1 ratio data were comparable during nighttime sleep following any experimental trial. However, according to the current study's findings, the water-based HIIE resulted in a greater nighttime dip. This finding may be particularly significant for hypertensive individuals classified as nondippers (Park et al., 2005). Scheduling water-based HIIE may enhance or even restore nighttime BP fall.

$\mathrm{HRV}$ analysis is widely accepted as a noninvasive technique for evaluating autonomic influences on the heart (Task Force of the European Society of Cardiology and the North American Society of Pacing and Electrophysiology, 1996). The autonomic nervous system's complex function, the relationship between parasympathetic and sympathetic branches, and their contributions to cardiac regulation during recovery remain unknown. One of our objectives was to compare postexercise HRV recovery following the water- and land-based HIIEs performed at comparable workloads; accordingly, we hypothesized that HRV recovery would be slower post land-based HIIE.

The reductions of $\ln \mathrm{HF}$ and RMSSD in the land-based HIIE during the nighttime indicate that vagal activity has not yet fully recovered. Reduced lnHF and RMSSD values have been reported to be associated with fatigue symptoms (Escorihuela et al., 2020). Moreover, the increase of the LF/HF ratio during the daytime reflected a reduction in vagal activity. Therefore, the land-based HIIE may require more than 24-hr of rest to restore cardiac autonomic balance. As for the water-based HIIE, the daytime HRV parameters were not different compared to the no-exercise control condition, indicating fully restored cardiac autonomic activity. It is critical to note that in the water-based HIIE, HRV parameters are restored to resting levels within $24 \mathrm{hr}$ of recovery. This indicates that the parasympathetic reactivation in the water-based HIIE occurs faster than in the land-based HIIE. The precise mechanisms by which water-based exercises improve vagal tone remain unknown. However, angiotensin II and NO are potential mediators, as both 
are involved in postexercise hypotension. While angiotensin II is known to suppress cardiac vagal tone during and after exercise, the increased NO bioavailability during exercise training may increase vagal activity during recovery (Routledge et al., 2010).

Water-based exercise is becoming more popular and recommended among the obese and those with limited joint mobility (Vincent and Mathews, 2013). The current study was designed to investigate the affective and enjoyable responses associated with water-based and land-based HIIE. As hypothesized, the waterbased HIIE was perceived as more affective and enjoyable. The water-based HIIE resulted in greater affective motivation to continue exercising in the final three sets, as well as a greater enjoyment of exercise in the final two sets. For the water-based HIIE program, increasing the number of exercise sets is feasible. Although the land-based HIIE has been shown to significantly reduce SBP in the first hour in prehypertensive obese adults, it may limit adherence and compliance due to the lower rate of affective response and enjoyability. In addition, the buoyancy associated with aquatic exercise minimizes joint impact during water-based HIIE, resulting in decreased stress on joints and muscles (Becker, 2009). Muscle soreness is greater following land-based, rather than water-based HIIE, as buoyancy supports bodyweight and alleviates stress on joints and muscles during exercise (Becker, 2009). What's more, the water-based HIIE program enables obese or overweight individuals to exercise safely.

The current study extends the clinical relevance of postexercise hypotension in water-based exercises by demonstrating that an aquatic environment may be a useful tool for prehypertensive obese individuals participating in exercise. As a nonpharmacological therapy, water-based HIIE can help with BP control, hypertension prevention, and cardiac autonomic restoration. Despite the significant contributions made by our findings, this study also presents certain limitations. We consider that the sample size should be increased. It is critical to emphasize, however, that all subjects completed all sessions which contributed to decreasing interference in the results. We did not directly measure $\mathrm{VO}_{2}$ to ensure that both HIIE sessions had comparable energy expenditure. Nevertheless, we were able to adjust the exercise intensity of the HIIE in the immersed state by implementing HR and RPE monitoring to ensure that it was equivalent to that of HIIE on dry land. Water temperature also affects BP, as demonstrated in older men performing upper-body aquatic exercises in various temperatures $\left(28^{\circ} \mathrm{C}\right.$ vs. $36^{\circ} \mathrm{C}$ ). SBP and DBP were lower in the warmer conditions, whereas HR was higher (Bergamin et al., 2015). Our water temperature was $30^{\circ} \mathrm{C}$, which is considered thermoneutral for wa- ter exercise (Christie et al., 1990).

We conclude that both HIIE modalities have the potential to result in expressive postexercise hypotension. Notably, following the water-based HIIE, SBP was reduced for $12 \mathrm{hr}$, with cardiac autonomic balance restored within $24 \mathrm{hr}$. These results suggest that water-based HIIE is more effective at reducing nighttime SBP while requiring less recovery time than land-based HIIE in prehypertensive obese men. Additionally, the water-based HIIE program was found to be more effectual and enjoyable in addition to assisting prehypertensive obese individuals to complete their exercise program.

\section{CONFLICT OF INTEREST}

No potential conflict of interest relevant to this article was reported.

\section{ACKNOWLEDGMENTS}

We wish to thank the participants for their enthusiastic participation throughout the study. The authors received no financial support for this article.

\section{REFERENCES}

Albert CM, Mittleman MA, Chae CU, Lee IM, Hennekens CH, Manson JE. Triggering of sudden death from cardiac causes by vigorous exertion. N Engl J Med 2000;343:1355-1361.

Angadi SS, Bhammar DM, Gaesser GA. Postexercise hypotension after continuous, aerobic interval, and sprint interval exercise. J Strength Cond Res 2015;29:2888-2893.

Barbosa TM, Marinho DA, Reis VM, Silva AJ, Bragada JA. Physiological assessment of head-out aquatic exercises in healthy subjects: a qualitative review. J Sports Sci Med 2009;8:179-189.

Becker BE. Aquatic therapy: scientific foundations and clinical rehabilitation applications. PM R 2009;1:859-872.

Bergamin M, Ermolao A, Matten S, Sieverdes JC, Zaccaria M. Metabolic and cardiovascular responses during aquatic exercise in water at different temperatures in older adults. Res Q Exerc Sport 2015;86:163-171.

Bocalini DS, Bergamin M, Evangelista AL, Rica RL, Pontes FL Jr, Figueira A Junior, Serra AJ, Rossi EM, Tucci PJF, Dos Santos L. Post-exercise hypotension and heart rate variability response after water- and landergometry exercise in hypertensive patients. PLoS One 2017;12:e0180216.

Calhoun DA, Harding SM. Sleep and hypertension. Chest 2010;138:434443. 
Christie JL, Sheldahl LM, Tristani FE, Wann LS, Sagar KB, Levandoski SG, Ptacin MJ, Sobocinski KA, Morris RD. Cardiovascular regulation during head-out water immersion exercise. J Appl Physiol 1990;69:657-664.

Davy KP, Hall JE. Obesity and hypertension: two epidemics or one? Am J Physiol Regul Integr Comp Physiol 2004;286:R803-813.

Donnelly JE, Blair SN, Jakicic JM, Manore MM, Rankin JW, Smith BK; American College of Sports Medicine. American College of Sports Medicine Position Stand. Appropriate physical activity intervention strategies for weight loss and prevention of weight regain for adults. Med Sci Sports Exerc 2009;41:459-471.

El Agaty SM, Kirmani A, Labban E. Heart rate variability analysis during immediate recovery from exercise in overweight/obese healthy young adult females. Ann Noninvasive Electrocardiol 2017;22:e12427.

Escorihuela RM, Capdevila L, Castro JR, Zaragozà MC, Maurel S, Alegre J, Castro-Marrero J. Reduced heart rate variability predicts fatigue severity in individuals with chronic fatigue syndrome/myalgic encephalomyelitis. J Transl Med 2020;18:4.

Fletcher GF, Balady GJ, Amsterdam EA, Chaitman B, Eckel R, Fleg J, Froelicher VF, Leon AS, Piña IL, Rodney R, Simons-Morton DA, Williams MA, Bazzarre T. Exercise standards for testing and training: a statement for healthcare professionals from the American Heart Association. Circulation 2001;104:1694-1740.

Gabrielsen A, Videbaek R, Johansen LB, Warberg J, Christensen NJ, Norsk P. Immediate baroreflex-neuroendocrine interactions in humans during graded water immersion. J Gravit Physiol 1996;3:22-23.

Gabrielsen A, Warberg J, Christensen NJ, Bie P, Stadeager C, Pump B, Norsk $\mathrm{P}$. Arterial pulse pressure and vasopressin release during graded water immersion in humans. Am J Physiol Regul Integr Comp Physiol 2000;278:R1583-1588.

Hardy CJ, Rejeski WJ. Not what, but how one feels: the measurement of affect during exercise. J Sport Exerc Psychol 1989;11:304-317.

Hawker GA, Mian S, Kendzerska T, French M. Measures of adult pain: Visual Analog Scale for Pain (VAS Pain), Numeric Rating Scale for Pain (NRS Pain), McGill Pain Questionnaire (MPQ), Short-Form McGill Pain Questionnaire (SF-MPQ), Chronic Pain Grade Scale (CPGS), Short Form-36 Bodily Pain Scale (SF-36 BPS), and Measure of Intermittent and Constant Osteoarthritis Pain (ICOAP). Arthritis Care Res (Hoboken) 2011;63 Suppl 11:S240-252.

Hecksteden A, Grütters T, Meyer T. Association between postexercise hypotension and long-term training-induced blood pressure reduction: a pilot study. Clin J Sport Med 2013;23:58-63.

Jimenez C, Regnard J, Robinet C, Mourot L, Gomez-Merino D, Chennaoui M, Jammes Y, Dumoulin G, Desruelle AV, Melin B. Whole body immersion and hydromineral homeostasis: effect of water temperature. Eur J Appl Physiol 2010;108:49-58.
Jones H, George K, Edwards B, Atkinson G. Exercise intensity and blood pressure during sleep. Int J Sports Med 2009;30:94-99.

Keating SE, Coombes JS, Stowasser M, Bailey TG. The role of exercise in patients with obesity and hypertension. Curr Hypertens Rep 2020; 22:77.

Leggio M, Lombardi M, Caldarone E, Severi P, D'emidio S, Armeni M, Bravi V, Bendini MG, Mazza A. The relationship between obesity and hypertension: an updated comprehensive overview on vicious twins. Hypertens Res 2017;40:947-963.

Liu S, Goodman J, Nolan R, Lacombe S, Thomas SG. Blood pressure responses to acute and chronic exercise are related in prehypertension. Med Sci Sports Exerc 2012;44:1644-1652.

Lopera CA, da Silva DF, Bianchini JA, Locateli JC, Moreira AC, Dada RP, Thivel D, Nardo N Junior. Effect of water- versus land-based exercise training as a component of a multidisciplinary intervention program for overweight and obese adolescents. Physiol Behav 2016;165:365-373.

Lopes WA, Matos FO, Porto FE. Is obesity related to postexercise cardiac autonomic recovery? Ann Noninvasive Electrocardiol 2017;22:e12459.

MacDonald JR. Potential causes, mechanisms, and implications of post exercise hypotension. J Hum Hypertens 2002;16:225-236.

MacDonald JR, MacDougall JD, Hogben CD. The effects of exercise duration on post-exercise hypotension. J Hum Hypertens 2000;14:125-129.

Malliani A. Heart rate variability: from bench to bedside. Eur J Intern Med 2005;16:12-20.

Mancia G, Fagard R, Narkiewicz K, Redón J, Zanchetti A, Böhm M, Christiaens T, Cifkova R, De Backer G, Dominiczak A, Galderisi M, Grobbee DE, Jaarsma T, Kirchhof P, Kjeldsen SE, Laurent S, Manolis AJ, Nilsson PM, Ruilope LM, Schmieder RE, Sirnes PA, Sleight P, Viigimaa M, Waeber B, Zannad F; Task Force Members. 2013 ESH/ESC Guidelines for the management of arterial hypertension: the Task Force for the management of arterial hypertension of the European Society of Hypertension (ESH) and of the European Society of Cardiology (ESC). J Hypertens 2013;31:1281-1357.

Marçal IR, Goessler KF, Buys R, Casonatto J, Ciolac EG, Cornelissen VA. Post-exercise hypotension following a single bout of high intensity interval exercise vs. a single bout of moderate intensity continuous exercise in adults with or without hypertension: a systematic review and meta-analysis of randomized clinical trials. Front Physiol 2021;12: 675289.

Meredith-Jones K, Waters D, Legge M, Jones L. Upright water-based exercise to improve cardiovascular and metabolic health: a qualitative review. Complement Ther Med 2011;19:93-103.

Park S, Jastremski CA, Wallace JP. Time of day for exercise on blood pressure reduction in dipping and nondipping hypertension. J Hum Hypertens 2005;19:597-605. 
Pescatello LS, Franklin BA, Fagard R, Farquhar WB, Kelley GA, Ray CA; American College of Sports Medicine. American College of Sports Medicine position stand. Exercise and hypertension. Med Sci Sports Exerc 2004;36:533-553.

Pescatello LS, MacDonald HV, Ash GI, Lamberti LM, Farquhar WB, Arena R, Johnson BT. Assessing the existing professional exercise recommendations for hypertension: a review and recommendations for future research priorities. Mayo Clin Proc 2015a;90:801-812.

Pescatello LS, MacDonald HV, Lamberti L, Johnson BT. Exercise for hypertension: a prescription update integrating existing recommendations with emerging research. Curr Hypertens Rep 2015b;17:87.

Pickering TG, Hall JE, Appel LJ, Falkner BE, Graves J, Hill MN, Jones DW, Kurtz T, Sheps SG, Roccella EJ. Recommendations for blood pressure measurement in humans and experimental animals: part 1: blood pressure measurement in humans: a statement for professionals from the Subcommittee of Professional and Public Education of the American Heart Association Council on High Blood Pressure Research. Circulation 2005;111:697-716.

Pimenta FC, Montrezol FT, Dourado VZ, da Silva LFM, Borba GA, de Oliveira Vieira W, Medeiros A. High-intensity interval exercise promotes post-exercise hypotension of greater magnitude compared to moderate-intensity continuous exercise. Eur J Appl Physiol 2019;119:12351243.

Pober DM, Braun B, Freedson PS. Effects of a single bout of exercise on resting heart rate variability. Med Sci Sports Exerc 2004;36:1140-1148.

Ramirez-Jimenez M, Morales-Palomo F, Pallares JG, Mora-Rodriguez R, Ortega JF. Ambulatory blood pressure response to a bout of HIIT in metabolic syndrome patients. Eur J Appl Physiol 2017;117:1403-1411.

Reilly T, Dowzer CN, Cable NT. The physiology of deep-water running. J
Sports Sci 2003;21:959-972.

Rodriguez D, Silva V, Prestes J, Rica RL, Serra AJ, Bocalini DS, Pontes FL Jr. Hypotensive response after water-walking and land-walking exercise sessions in healthy trained and untrained women. Int J Gen Med 2011;4:549-554.

Routledge FS, Campbell TS, McFetridge-Durdle JA, Bacon SL. Improvements in heart rate variability with exercise therapy. Can J Cardiol 2010;26:303-312.

Sosner P, Gayda M, Dupuy O, Garzon M, Lemasson C, Gremeaux V, Lalongé J, Gonzales M, Hayami D, Juneau M, Nigam A, Bosquet L. Ambulatory blood pressure reduction following high-intensity interval exercise performed in water or dryland condition. J Am Soc Hypertens 2016;10:420-428.

Stanley DM, Cumming J. Are we having fun yet? Testing the effects of imagery use on the affective and enjoyment responses to acute moderate exercise. Psychol Sport Exerc 2010;11:582-590.

Stanley J, Peake JM, Buchheit M. Cardiac parasympathetic reactivation following exercise: implications for training prescription. Sports Med 2013;43:1259-1277.

Task Force of the European Society of Cardiology and the North American Society of Pacing and Electrophysiology. Heart rate variability: standards of measurement, physiological interpretation and clinical use. Circulation 1996;93:1043-1065.

Tucker WJ, Angadi SS, Gaesser GA. Excess postexercise oxygen consumption after high-intensity and sprint interval exercise, and continuous steady-state exercise. J Strength Cond Res 2016;30:3090-3097.

Vincent HK, Mathews A. Obesity and mobility in advancing age: mechanisms and interventions to preserve independent mobility. Curr Obes Rep 2013;2:275-283. 